\title{
Unlocking the secrets of IgA
}

Two new studies have shed light on the role of IgA in maintaining gut microbiota homeostasis. Using a mouse model, Gregory Donaldson, Sarkis Mazmanian and colleagues demonstrated that IgA is required for commensal bacterial species to colonize specific mucosal niches. Complementing these results, Jehane Fadlallah, Martin Larsen, Guy Gorochov and colleagues found that IgA deficiency in humans is associated with gut dysbiosis, notably the loss of beneficial commensal bacteria.

The gut mucosal immune system is a key regulator of interactions between the host and the intestinal microbiota. Gut homeostasis relies upon the maintenance of the intestinal barrier and the prevention of aberrant immune activation by commensal microorganisms. Evidence from human studies and animal models suggests that IgA, the predominant antibody isotype secreted in the gut, is able to alter the composition of the gut microbiota and modulate infection susceptibility. Moreover, the evolutionarily conserved nature of very high gut IgA secretion (several grams per day in humans) supports

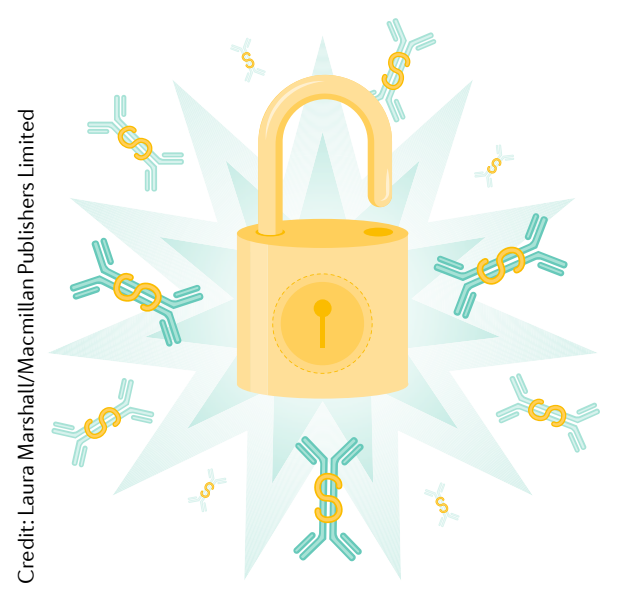

the rationale that the protein has important functions. However, the exact roles of secreted IgA and the mechanisms by which it modulates gut microbiota composition are unclear.

Building on findings from a previous paper that identified bacterial genes involved in bacterial colonization of intestinal crypts,

Donaldson and colleagues engineered bacteria to lack these crypt colonization genes and characterized the resulting phenotype. Unexpectedly, this research led them to investigate how IgA influences mucosal colonization. "As we explored this question, we made several unexpected discoveries along the way: first, that a primary function of these colonization genes was regulation of bacterial capsular polysaccharides and, second, that this regulation was modulating binding by IgA in the gut," explains Donaldson.

"The biggest finding here is that IgA can have a positive effect on bacteria by promoting mucosal colonization," notes Donaldson. "From a microbiology perspective, what is also interesting is that this was mediated by capsular polysaccharides, which have been traditionally regarded as virulence factors." These novel findings open up new avenues for research to investigate how IgA responses differ between bacterial species or strains, and the functional implications of these potentially different responses.

In a separate paper published in Science Translational Medicine, Gorochov and colleagues used a combined flow cytometry and metagenomics approach to explore how IgA levels affect the gut microbiota composition in patients with selective IgA deficiency.

"To put it bluntly: the main finding is that $\operatorname{Ig} \mathrm{A}$ is there in the gut not only to protect against bad bugs (pathobionts and pathogens), but also to protect good bugs (beneficial commensals)," says Gorochov. "Another important finding is the demonstration that IgM can compensate for IgA (in IgA-deficient patients), but not completely," he adds. Notably, although IgM was able to bind many Gram-positive bacteria such as Bifidobacterium and Faecalibacterium, there was less efficient IgM binding to Enterobacteriaceae or Prevotellaceae families, both of which are associated with proinflammatory effects and are over-represented in patients with selective IgA deficiency.

In sum, these findings are compatible with those of Donaldson and colleagues, and have ramifications for future work, Gorochov says. "Some patients with common variable immune deficiency suffer from severe dysbiosis, and ultimately succumb from nonalcoholic cirrhosis," explains Gorochov. "The metabolic pathways involved are unknown and should be now explored. From an interventions standpoint, we believe our results pave the way for clinical trials based on oral IgA supplementation, obviously in immunoglobulin deficiencies, but possibly in many other dysbiosis-associated conditions such as IBD."

Hugh Thomas

ORIGINAL ARTICLES Donaldson, G. P. et al. Gut microbiota utilize immunoglobulin A for mucosal colonization. Science 360, 795-800 (2018)| Fadlallah, J. et al. Microbial ecology perturbation in human IgA deficiency. Sci. Transl. Med. 10, eaan1217 (2018) 